Proceedings of the

International Geometry Center

Vol. 14, no. 3 (2021) pp. 164-186

\title{
On homotopy nilpotency of some suspended spaces
}

\author{
Marek Golasiński
}

\begin{abstract}
A homological criterium from [14] is applied to investigate the homotopy nilpotency of some suspended spaces. We investigate the homotopy nilpotency of the wedge sum and smash products of Moore spaces $M(A, n)$ with $n \geqslant 1$. The homotopy nilpotency of homological spheres are studied as well.
\end{abstract}

Анотація. В роботі використовується гомологічний критерій з [14] для дослідження гомотопічної нільпотентності надбудов деякого класу просторів. Досліджується гомотопічна нільпотентність букетів та смеш-добутків просторів Мура $M(A, n), n \geqslant 1$. Також вивчається гомотопічна нільпотентність гомологічних сфер.

\section{INTRODUCTION}

In the group theory, if we consider only nilpotent groups, the nilpotency class is the one which measures a distance from commutativity. Already G. Whitehead [26] had the insight that the (J.H.C.) Whitehead products satisfy identities which reflect commutator identities for groups. Berstein and Ganea [3] adapted the nilpotency to $H$-spaces as follows. Let $X$ be an $H$-space, $\varphi_{X, 1}=\mathrm{id}_{X}$, and $\varphi_{X, 2}: X^{2} \rightarrow X$ the commutator map. Put $\varphi_{X, n+1}=\varphi_{X, 1} \circ\left(\operatorname{id}_{X} \times \varphi_{X, n}\right)$ for $(n+1)$-fold commutator map of $X$ with $n \geqslant 2$. An $H$-space $X$ is called homotopy nilpotent of class $n$ if $\varphi_{X, n+1} \simeq *$, is nullhomotopic but $\varphi_{X, n}$ is not, [3]. In this case, we write nil $X=n$.

2010 Mathematics Subject Classification: Primary: 54E30, secondary: 20F18, 55P15.

Keywords: abelian group, cartesian product, homology sphere, nilpotency class, $n$-fold commutator map, $H$-space, loop space, Moore space, localization, Samelson product, smash product, suspension space, wedge sum, Whitehead product

Ключові слова: абелева група, декартовий добуток, гомологічна сфера, клас нільпотентності, відображення $n$-кратного комутування, $H$-простір, простір петель, простір Мура, локалізація, добуток Самельсона, смеш-добуток, надбудова, букет просторів, добуток Уайтхеда

DOI: http://dx.doi.org/10.15673/tmgc.v14i3.2032 
Then, Berstein and Ganea [3] introduced a concept of the homotopy nilpotency of a pointed space by means of its loop space. In particular, the $m$-iterated Samelson products vanish in the loop space $\Omega(X)$, or equivalently, the $m$-iterated Whitehead products vanish in $X$ provided $m>$ nil $\Omega(X)$. We refer to [27, Chapter X] for details on Samelson and Whitehead products.

The homotopy nilpotency classes nil $X$ of associative $H$-spaces $X$ has been extensively studied as well as their homotopy commutativity. Work of Hopkins [17] drew renewed attention to such problems by relating this classical nilpotency notion with the nilpotence theorem of Devinatz, Hopkins, and Smith [6]. Although many results on the homotopy nilpotency have been obtained, the homotopy nilpotency classes have been determined in very few cases.

It is well-known that for the loop space $\Omega\left(\mathbb{S}^{m}\right)$ of the $m$-sphere $\mathbb{S}^{m}$ we have nil $\Omega\left(\mathbb{S}^{m}\right)=1$ if and only if $m=1,3,7$ and

$$
\operatorname{nil} \Omega\left(\mathbb{S}^{m}\right)= \begin{cases}2 & \text { for odd } m \text { and } m \neq 1,3,7 \text { or } m=2, \\ 3 & \text { for even } m \geqslant 4 .\end{cases}
$$

Write $\mathbb{K} P^{m}$ for the projective $m$-space for $\mathbb{K}=\mathbb{R}, \mathbb{C}$, the field of reals or complex numbers and $\mathbb{H}$ for the skew $\mathbb{R}$-algebra of quaternions. Then, the homotopy nilpotency of $\Omega\left(\mathbb{K} P^{m}\right)$ has been first studied by Ganea [10], Snaith [24] and then their $p$-localization $\Omega\left(\left(\mathbb{K} P^{m}\right)_{(p)}\right)$ by Meier [19]. The homotopy nilpotency of the loop spaces of Grassmann and Stiefel manifolds, and their $p$-localization have been extensively studied in [13].

Let $\mathbb{S}_{(p)}^{2 m-1}$ be the $p$-localization of the sphere $\mathbb{S}^{2 m-1}$ at a prime $p$. The main result of the paper [12] is the explicit determination of the homotopy nilpotence class of a wide range of homotopy associative multiplications on localized spheres $\mathbb{S}_{(p)}^{2 m-1}$ for $p>3$. Furthermore, the paper [11] reviews known and states some new results on the homotopy nilpotency and conilpotency of spaces.

The article [14] grew out of our desire to develop techniques in the study of the homotopy nilpotency classes of Moore spaces $M(A, n)$ for $n \geqslant 1$. In this partially survey paper [14, Proposition 3.2] is applied to investigate the homotopy nilpotency of the wedge sum and smash products of Moore spaces $M(A, n)$ with $n \geqslant 1$ and the homotopy nilpotency of homological spheres as well. To have kept all results together, we decided to include the main part of [14] here.

In Section 1 we set stages for developments to come. This introductory section is devoted to a general discussion and establishes notations on the homotopy nilpotency of $H$-spaces used in the rest of the paper. Given a space $X$, we consider the iterated Samelson product $s_{k}: X^{\wedge k} \rightarrow \Omega \Sigma(X)$ to 
show in Proposition 1.11 (see [14, Proposition 3.2]) that the space $\Omega \Sigma(X)$ is not homotopy nilpotent provided the homology $\tilde{H}_{*}(X, \mathbb{F})$ has at least two primitive generators, where $\mathbb{F}$ is a field.

Section 2 first takes up the systematic study of the homotopy nilpotency of Moore spaces $M(A, n)$ for $n \geqslant 1$ already presented in [14]. Then, we generalize [14, Theorem 3.8] and state the main result

Theorem 2.5. Let $m \geqslant 1, n_{1}, \ldots, n_{m} \geqslant 2$ and $M\left(A_{k}, n_{k}\right)$ be Moore spaces of type $\left(A_{k}, n_{k}\right)$ for $k=1, \ldots, m$. Then:

(1) nil $\Omega\left(\left(M\left(A_{1}, n_{1}\right) \times \cdots \times\left(M\left(A_{m}, n_{m}\right)\right)<\infty\right.\right.$ if and only if $A_{k}$ are torsionfree groups with rank $\mathrm{r}\left(A_{k}\right)=1$ for $k=1, \ldots, m$;

(2) nil $\Omega\left(\left(M\left(A_{1}, n_{1}\right) \vee \cdots \vee M\left(A_{m}, n_{m}\right)\right)<\infty\right.$ if and only if $m=1$ and

$A_{1}$ is a torsion-free group with rank $\mathrm{r}\left(A_{1}\right)=1$.

Unfortunately, Moore spaces of type $(A, 1)$ are not determined uniquely (up to homotopy) by an abelian group $A$. We present constructions and analyse the homotopy nilpotency of some such spaces and their wedge sums.

Next, we study the homotopy nilpotency of the smash products of Moore spaces and state

Proposition 2.8. If $m, n \geqslant 1$ then

$$
\operatorname{nil} \Omega(M(A, m) \wedge M(B, n))<\infty
$$

if and only if the group $A \otimes B$ is torsion free with the rank $\mathrm{r}(A \otimes B) \leqslant 1$ and $\operatorname{Tor}(A, B)=0$.

At the end, the homotopy nilpotency of homology spheres and their suspensions are taken into account as well.

\section{Prerequisites}

All spaces and maps in this note are assumed to be connected and based with the homotopy type of $C W$-complexes unless we assume otherwise. We also do not distinguish notationally between a continuous map and its homotopy class. We write $\Omega(X)$ (resp. $\Sigma(X))$ for the loop (resp. suspension) space on a space $X$ and $[X, Y]$ for the set of homotopy classes of maps $X \rightarrow Y$.

Given a space $X$, we use the customary notations $X \vee X$ and $X \wedge X$ for the wedge and the smash square of $X$, respectively. Recall that an $H$-space is a pair $(X, \mu)$, where $X$ is a space and $\mu: X \times X \rightarrow X$ is a map such that the diagram

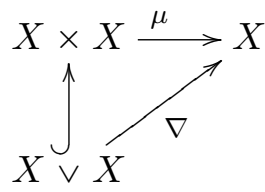


commutes up to homotopy, where $\nabla: X \vee X \rightarrow X$ is the folding map. We call $\mu$ a multiplication or an $H$-structure for $X$. Two examples of $H$-spaces come in mind: topological groups and the loop spaces $\Omega(X)$. In the sequel, we identify an $H$-space $(X, \mu)$ with the space $X$.

An $H$-space $X$ is called a group-like space if $X$ satisfies all the axioms of groups up to homotopy. Recall that a homotopy associative $H-C W$ complex always has a homotopy inverse. More precisely, according to [28, 1.3.2. Corollary] (see also [1, Proposition 8.4.4]), we have

Proposition 1.1. If $X$ is a homotopy associative $H$-CW-complex then $X$ is a group-like space.

From now on, we assume that any $H$-space $X$ is group-like.

Given spaces $X_{1}, \ldots, X_{n}$, we use the customary notations $X_{1} \times \cdots \times$ $X_{n}$ for their Cartesian product and $T_{m}\left(X_{1}, \ldots, X_{n}\right)$ for the subspace of $X_{1} \times \cdots \times X_{n}$ consisting of those points with at least $m$ coordinates at base points with $m=0,1, \ldots, n$. Then, $T_{0}\left(X_{1}, \ldots, X_{n}\right)=X_{1} \times \cdots \times X_{n}$, $T_{1}\left(X_{1}, \ldots, X_{n}\right)$ is the so called the fat wedge of spaces $X_{1}, \ldots, X_{n}$ and $T_{n-1}\left(X_{1}, \ldots, X_{n}\right)=X_{1} \vee \cdots \vee X_{n}$, the wedge sum of spaces $X_{1}, \ldots, X_{n}$. We write

$$
j_{m}\left(X_{1}, \ldots, X_{n}\right): T_{m}\left(X_{1}, \ldots, X_{n}\right) \rightarrow X_{1} \times \cdots \times X_{n}
$$

for the inclusion map with $m=0,1, \ldots, n$ and

$$
X_{1} \wedge \cdots \wedge X_{n}=X_{1} \times \cdots \times X_{n} / T_{1}\left(X_{1}, \ldots, X_{n}\right)
$$

for the smash product of spaces $X_{1}, \ldots, X_{n}$.

Let $f_{m}:\left(X_{m}, \star_{m}\right) \rightarrow\left(Y_{m}, *_{m}\right)$ be continuous maps of pointed topological spaces for $m=1, \ldots, n$. The map

$$
f_{1} \times \cdots \times f_{n}:\left(X_{1} \times \cdots \times X_{n},\left(\star_{1}, \ldots, \star_{n}\right)\right) \rightarrow\left(Y_{1} \times \cdots \times Y_{n},\left(*_{1}, \ldots, *_{n}\right)\right)
$$

sends each point $\left(x_{1}, \ldots, x_{n}\right) \in X_{1} \times \cdots \times X_{n}$ to $\left(f_{1}\left(x_{1}\right), \ldots, f_{n}\left(x_{n}\right)\right)$ and restricts to maps

$$
T_{m}\left(f_{1}, \ldots, f_{n}\right): T_{m}\left(X_{1}, \ldots, X_{n}\right) \rightarrow T_{m}\left(Y_{1}, \ldots, Y_{n}\right)
$$

with $m=0,1, \ldots, n$. If $X_{m}=X$ and $f_{m}=f$ for $m=1, \ldots, n$ then we write

$$
\begin{aligned}
X^{n} & =X_{1} \times \cdots \times X_{n}, & X^{\wedge n} & =X_{1} \wedge \cdots \wedge X_{n}, \\
f^{n} & =f_{1} \times \cdots \times f_{n}, & f^{\wedge n} & =f_{1} \wedge \cdots \wedge f_{n} .
\end{aligned}
$$

The identity map of a space $X$ involved is consistently denoted by $\iota_{X}$.

Given an $H$-group $X$, the functor $[-, X]$ takes its values in the category of groups. One may then ask when those functors take their values in 
various subcategories of groups. For example, $X$ is homotopy commutative if and only if $[Y, X]$ is abelian for all $Y$.

Given an $H$-space $X$, we write $\varphi_{X, 1}=\iota_{X}, \varphi_{X, 2}: X^{2} \rightarrow X$ for the basic commutator map and $\varphi_{X, n+1}=\varphi_{X, 2} \circ\left(\varphi_{X, n} \times \iota_{X}\right)$ for $n \geqslant 2$.

The nilpotency class. The nilpotency class nil $(X, \mu)$ of an $H$-space $(X, \mu)$ is the least integer $n \geqslant 0$ for which the map $\varphi_{X, n+1} \simeq *$ is nullhomotopic and we call the homotopy associative $H$-space $X$ homotopy nilpotent. If no such integer exists, we put nil $(X, \mu)=\infty$. In the sequel, we simply write nil $X$ for the nilpotency class of an $H$-space $X$. Thus, nil $X=0$ if and only if $X$ is contractible and, as is easily seen, nil $X \leqslant 1$ if and only if $X$ is homotopy commutative.

It is obvious that for homotopy nilpotent group-like spaces $X_{1}, \ldots, X_{m}$, we have

$$
\operatorname{nil}\left(X_{1} \times \cdots \times X_{m}\right)=\max \left\{\operatorname{nil} X_{1}, \ldots, \operatorname{nil} X_{m}\right\} .
$$

The set $\pi_{0}(X)$ of all path-components of an $H$-space $X$ is known to be a group. The following result is easy to prove

Lemma 1.2. If $X$ is an $H$-space and the path component of the base-point $\star \in X$ is contractible then nil $\pi_{0}(X)=$ nil $X$.

The definition of the nilpotency classes may be extended to maps. The nilpotency class nil $f$ of an $H$-map $f: X_{1} \rightarrow X_{2}$ is the least integer $n \geqslant 0$ for which the map $f \circ \varphi_{X, n+1}: X_{1}^{n+1} \rightarrow X_{2}$ is nullhomotopic. If no such integer exists, we put nil $f=\infty$.

In the sequel we need the following

Lemma 1.3. If $X$ is an $H$-space then the composite map

$$
T_{1}(X, \ldots, X) \stackrel{j_{1}(X, \ldots, X)}{\longrightarrow} X^{n} \stackrel{\varphi_{X, n}}{\longrightarrow} X
$$

is nullhomotopic.

Since the space $X^{\wedge n}$, the $n$-th smash power of $X$, is the homotopy cofiber of the map

$$
j_{1}(X, \ldots, X): T_{1}(X, \ldots, X) \rightarrow X^{n},
$$

the result above implies an existence of a map $\bar{\varphi}_{X, n}: X^{\wedge} n \rightarrow X$ for $n \geqslant 1$ with $\bar{\varphi}_{1, X}=\varphi_{X, 1}$.

It is well known that the quotient map $X^{n} \rightarrow X^{\wedge n}$ has a right homotopy inverse after suspending for $n \geqslant 1$, and the fact that $X$ is an $H$-space means that the suspension map $[Y, X] \rightarrow[\Sigma Y, \Sigma X]$ is a monomorphism for any space $Y$. Thus, we may state 
Proposition 1.4. Let $X$ be an $H$-space. Then $\varphi_{X, n} \simeq *$ if and only if $\bar{\varphi}_{X, n} \simeq *$ for $n \geqslant 1$.

Hence [3, 2.7. Theorem] and Proposition 1.4 lead to

Theorem 1.5. If $X$ is an $H$-space then

$$
\operatorname{nil} X=\sup _{m} \operatorname{nil}\left[X^{m}, X\right]=\sup _{m} \operatorname{nil}\left[X^{\wedge m}, X\right]=\sup _{Y} \operatorname{nil}[Y, X]
$$

where $m$ ranges over all integers and $Y$ over all topological spaces.

Furthermore, in view of [28, Lemma 2.6.1], we may state

Corollary 1.6. A connected $H$-space $X$ is homotopy nilpotent if and only if the functor $[-, X]$ on the category of all spaces is nilpotent group valued.

Proof. Certainly, the homotopy nilpotency of a connected associative $H$ space $X$ implies that the functor $[-, X]$ on the category of all pointed spaces is nilpotent group valued.

Now, suppose that the functor $[-, X]$ is nilpotent groups valued and nil $\left[\prod_{1}^{\infty} X, X\right]<n$. Then, for the projection map $\prod_{1}^{\infty} X \rightarrow X^{n}$ on the first $n$ factors, the composite map

$$
\prod_{1}^{\infty} X \rightarrow X^{n} \stackrel{\varphi_{X, n}}{\longrightarrow} X
$$

is nullhomotopic. Since, the projection $\prod_{1}^{\infty} X \rightarrow X^{n}$ has a retraction, we deduce that the map $\varphi_{X, n}: X^{n} \rightarrow X$ is also nullhomotopic and the proof is complete.

Further, notice that for a map $f: X \rightarrow Y$ of $H$-spaces, we have the commutative (up to homotopy) diagram

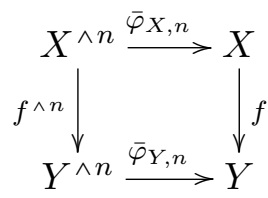

with $n \geqslant 1$. This yields

Remark 1.7. If $X^{\prime}$ is an $H$-subspace of an $H$-space $X$ and $r: X \rightarrow X^{\prime}$ its a homotopy $H$-retract then one can easily derive from the above that

$$
\operatorname{nil} X^{\prime} \leqslant \operatorname{nil} X
$$


Homotopy nilpotency of spaces. With any based path-connected space $X$, we associate the integer nil $\Omega(X)$ called the nilpotency class of $X$. Evidently, nil $\pi_{1}(X) \leqslant \operatorname{nil} \Omega(X)$. We give an extension of this result involving Whitehead products, generally denoted by $\left[\alpha_{1}, \alpha_{2}\right] \in \pi_{m_{1}+m_{2}-1}(X)$ if $\alpha_{i} \in \pi_{m_{i}}(X)$ for $m_{i} \geqslant 1$ with $i=1,2$. Define $(n+1)$-fold Whitehead products $\left[\alpha_{1}, \ldots, \alpha_{n+1}\right]$ as $\left[\left[\alpha_{1}, \ldots, \alpha_{n}\right], \alpha_{n+1}\right]$ if $\alpha_{i} \in \pi_{m_{i}}(X)$ for $m_{i} \geqslant 1$ with $i=1, \ldots, n+1$ agreeing that, for $n=0,[\alpha]=\alpha$.

Recall that W-length $X$, the Whitehead length of a space $X$ is the least integer $n \geqslant 0$ such that $\left[\alpha_{1}, \ldots, \alpha_{n+1}\right]=0$ for all $\alpha_{i} \in \pi_{m_{i}}(X), m_{i} \geqslant 1$; if no such integer exists, we put $\mathrm{W}$-length $X=\infty$.

Then, according to $[3,4.6$. Theorem], we have

Theorem 1.8. W-length $X \leqslant \operatorname{nil} \Omega(X)$.

Example 1.9. (1) It is well-known that

$$
\text { W-length } \mathbb{S}^{n}=\operatorname{nil} \Omega\left(\mathbb{S}^{n}\right)= \begin{cases}3, & \text { for } n \text { even with } n \neq 2, \\ 2, & \text { for } n \text { odd with } n \neq 1,3,7 \text { or } n=2, \\ 1, & \text { for } n=1,3,7 .\end{cases}
$$

(2) For the wedge $\mathbb{S}^{m} \vee \mathbb{S}^{n}$ of two spheres with $m, n \geqslant 2$, there is an iterated nontrivial Whitehead product of any length. Therefore, by Theorem 1.8 , we conclude that

$$
\operatorname{nil} \Omega\left(\mathbb{S}^{m} \vee \mathbb{S}^{n}\right)=\infty .
$$

The concept of a nilpotent space is due to Dror [8]. Recall that a pointed path-connected space $X$ is said to be nilpotent if its fundamental group $\pi_{1}(X)$ acts nilpotently on the higher homotopy groups $\pi_{n}(X)$ for $n \geqslant 1$. But, the action of $\pi_{1}(X)$ on $\pi_{n}(X)$ for $n \geqslant 1$ may be written in terms of Whitehead products. Therefore, the nilpotency of a space $X$ is a lower bound of its homotopy nilpotency nil $\Omega(X)$. Hence, by Theorem 1.8, the space $X$ is nilpotent if $\Omega(X)$ is homotopy nilpotent. But, by Example 1.9(2), not every space $\Omega(X)$ is homotopy nilpotent if $X$ is nilpotent or even simply connected.

Dror [8] has also published a far-reaching generalization of a classical theorem of J. H. C. Whitehead useful in the next section.

Theorem 1.10. If $f: X \rightarrow Y$ is a map of connected, pointed, $C W$ complexes which induces an isomorphism on integral homology, and if $X$ and $Y$ are nilpotent spaces, then $f$ is a homotopy equivalence.

To formulate the next result needed in the next section, let us fix first some notations and recall a definition. Given a pointed space $X$, write 
$i_{1}, i_{2}: X \rightarrow X \times X$ for the canonical embedding maps and $\Delta: X \rightarrow X \times X$ for the diagonal map. If $H_{m}(X, A)$ is the $m$-th homology group of $X$ with coefficient in an abelian group $A$ then an element $\alpha \in H_{m}(X, A)$ is said to be primitive if $\Delta_{*}(\alpha)=i_{1 *}(\alpha)+i_{2 *}(\alpha)$ for the induced homomorphisms

$$
i_{1 *}, i_{2 *}, \Delta_{*}: H_{m}(X, A) \rightarrow H_{m}(X \times X, A) .
$$

Now, we show that the space $\Omega \Sigma(X)$ is not homotopy nilpotent provided the homology $H_{*}(X, \mathbb{F})$ has at least two primitive generators, where $\mathbb{F}$ is a field.

Proposition 1.11. If $\tilde{H}_{*}(X, \mathbb{F})$ has at least two primitive generators, where $\mathbb{F}$ is a field then $\Omega \Sigma(X)$ is not homotopy nilpotent.

Proof. To see this, take homology $H_{*}(X, \mathbb{F})$ with $\mathbb{F}$-coefficients and recall that the Bott-Samelson theorem, [4], states that

$$
H_{*}(\Omega \Sigma X, \mathbb{F}) \approx T\left(\tilde{H}_{*}(X, \mathbb{F})\right),
$$

the tensor algebra on $\tilde{H}_{*}(X, \mathbb{F})$. This may be rewritten as $U L\left\langle\tilde{H}_{*}(X, \mathbb{F})\right\rangle$, where $L\left\langle\tilde{H}_{*}(X, \mathbb{F})\right\rangle$ is the free Lie algebra generated by $\tilde{H}_{*}(X, \mathbb{F})$ and $U L\left\langle\tilde{H}_{*}(X, \mathbb{F})\right\rangle$ is its universal enveloping algebra. Further, the suspension $E: X \rightarrow \Omega \Sigma X$ induces the inclusion of the generating set in homology. Now, consider the iterated Samelson product

$$
s_{k}: X^{\wedge k} \longrightarrow \Omega \Sigma(X),
$$

where $s_{1}=E, s_{k+1}=\left\langle E, s_{k}\right\rangle$, the Samelson product of $s_{k}$ and $E$ for $n \geqslant 2$. For any $x \in \tilde{H}_{*}(X)$ we have $E_{*}(x)=x$.

Recall that the effect of the Samelson product map on homology classes of loop spaces is presented e.g., in [27, Chapter X, Section 6]. If $x_{1}, \ldots, x_{k} \in$ $\tilde{H}_{*}(X, \mathbb{F})$ are primitive then the class

$$
x_{1} \otimes \cdots \otimes x_{k} \in \tilde{H}_{*}(X, \mathbb{F})^{\otimes k} \approx \tilde{H}_{*}\left(X^{\wedge k}, \mathbb{F}\right)
$$

is sent by $\left(s_{k}\right)_{*}$ to the iterated bracket $\left[x_{1},\left[x_{2}, \ldots\left[x_{k-1}, x_{k}\right]\right] \ldots\right]$. In particular, if $x, y \in \tilde{H}_{*}(X, \mathbb{F})$ are distinct primitive generators then the class

$$
[x,[x, \ldots[x, y]] \ldots] \in U L \tilde{H}_{*}(X, \mathbb{F}) \approx \tilde{H}_{*}(\Omega \Sigma(X), \mathbb{F})
$$

is in the image of $\left(s_{k}\right)_{*}$. Hence, $s_{k}$ cannot be nullhomotopic.

Notice that for the map $s_{k}: X^{\wedge k} \longrightarrow \Omega \Sigma(X)$ defined above, there is the factorization

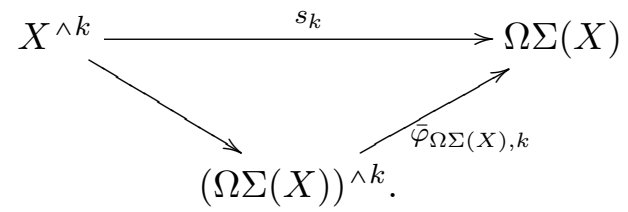


Consequently, the map $\bar{\varphi}_{\Omega \Sigma X, k}$ is not nullhomotopic provided the map $s_{k}$ is so and the proof is complete.

Notice that, in view of (1.1) and

$$
\tilde{H}_{*}\left(X_{1} \vee \cdots \vee X_{m}, \mathbb{F}\right)=\tilde{H}_{*}\left(X_{1}, \mathbb{F}\right) \oplus \cdots \oplus \tilde{H}_{*}\left(X_{m}, \mathbb{F}\right)
$$

for a field $\mathbb{F}$, Proposition 1.11 yields to the following

Corollary 1.12. If $\tilde{H}_{*}\left(X_{k}, \mathbb{F}\right)$ has at least two primitive generators for some $k$ with $k=1, \ldots, m$ then

$$
\begin{aligned}
\operatorname{nil}\left(\Omega \Sigma\left(X_{1}\right) \times \cdots \times \Omega \Sigma\left(X_{m}\right)\right) & =\infty, \\
\operatorname{nil}\left(\Omega\left(\Sigma\left(X_{1}\right) \vee \cdots \vee \Sigma\left(X_{m}\right)\right)\right. & =\infty .
\end{aligned}
$$

\section{Moore SPACES}

We take up the systematic study of the homotopy nilpotency of Moore spaces $M(A, n)$ for $n \geqslant 1$.

In Eckmann-Hilton duality, Moore $C W$-complexes play the role of dual objects of Eilenberg-MacLane $C W$-complexes. Let $A$ be an abelian group and $n$ any integer $\geqslant 1$. A space $X$ (if one such exists) satisfying $\pi_{j}(X)=0$ for $j<n, \pi_{n}(X) \approx A$ and $H_{i}(X)=0$ for $i>n$ is known as a Moore space of type $(A, n)$, or simply an $M(A, n)$ space. By [21], it is known that a $C W$-complex Moore space $M(A, n)$ with $n \geqslant 2$ exists and, in view of [15, Example 4.34], the homotopy type of a Moore space $M(A, n)$ is uniquely determined by $A$ and $n \geqslant 2$. This implies that every Moore space $M(A, n)$ with $n \geqslant 3$, is the suspension $\Sigma M(A, n-1)$. Furthermore, in [2, Section 2], it was shown that also $M(A, 2)$ is a suspension $\Sigma L(A)$ for some $C W$-complex $L(A)$.

By means of [25, Proposition 1.1], there exists an $M(A, 1)$ space if and only if the homology $H_{2}(A, \mathbb{Z})=0$ for the ring $\mathbb{Z}$ of integers. Recall that, by $\left[20\right.$, Thorem 3], $H_{2}(A, \mathbb{Z})=0$ if and only if $A \otimes A$ coincides with its subgroup generated by the diagonal, $\{a \otimes a ; a \in A\}$.

It is well-known that any abelian group homomorphism yields a map

$$
\tilde{\varphi}: M\left(A_{1}, n\right) \rightarrow M\left(A_{2}, n\right)
$$

with $H_{n}(\tilde{\varphi})=\varphi$ provided $n \geqslant 2$. We shown such a result for $n=1$ as well.

Proposition 2.1. Let $X_{1}, X_{2}$ be Moore spaces of types $\left(A_{1}, 1\right),\left(A_{2}, 1\right)$ constructed in [25] and $\varphi: A_{1} \rightarrow A_{2}$ an abelian group homomorphism. Then, there is a map $\tilde{\varphi}: X_{1} \rightarrow X_{2}$ with $H_{1}(\tilde{\varphi})=\varphi$. If the spaces $X_{1}, X_{2}$ are nilpotent then $\tilde{\varphi}: X_{1} \rightarrow X_{2}$ is a homotopy equivalence.

In particular, if $X_{1}, X_{2}$ are Moore spaces of type $(A, 1)$ constructed in [25] then there is a map $\widetilde{\mathrm{id}_{A}}: X_{1} \rightarrow X_{2}$ with $H_{1}\left(\widetilde{\operatorname{id}_{A}}\right)=\operatorname{id}_{A}$. 
Proof. For any space $X$, let $S(X)$ denote the subgroup of $H_{2}(X, \mathbb{Z})$ consisting of the spherical homology classes. Next, given an abelian group $A$ with $H_{2}(A, \mathbb{Z})$, consider the Eilenberg-MacLane $C W$-complex $K(A, 1)$. Then, by [25],

$$
H_{2}\left(K(A, 1)^{(2)}, \mathbb{Z}\right)=S\left(K(A, 1)^{(2)}\right)
$$

for the 2-skeleton $K(A, 1)^{(2)}$ of $K(A, 1)$. Since $H_{2}\left(K(A, 1)^{(2)}, \mathbb{Z}\right)$ is a free abelian group with a basis $\left\{a_{\mu}\right\}_{\mu \in I}$, there exist a map $f_{\mu}: \mathbb{S}^{2} \rightarrow K(A, 1)^{(2)}$ such that $f_{\mu *}(\gamma)=a_{\mu}$ for

$$
\left(f_{\mu}\right)_{*}: H_{2}\left(\mathbb{S}^{2}, \mathbb{Z}\right) \rightarrow H_{2}\left(K(A, 1)^{(2)}, \mathbb{Z}\right)
$$

and a generator $\gamma \in \mathrm{H}_{2}\left(\mathbb{S}^{2}, \mathbb{Z}\right)$. The $C W$-complex $M(A, 1)$ is obtained by attaching 3-cells to $K(A, 1)^{(2)}$ by means of the maps $f_{\mu}: \mathbb{S}^{2} \rightarrow K(A, 1)^{(2)}$ for $\mu \in I$. Consequently, we have the pushout diagram

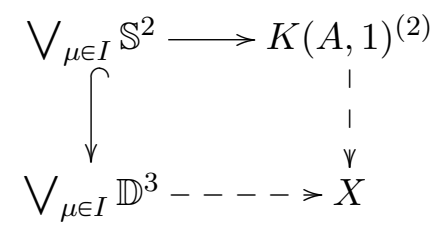

with $X$ a Moore space of type $(A, 1)$.

Now, given a group homomorphism $\varphi: A_{1} \rightarrow A_{2}$, the induced cellular map $\bar{\varphi}: K\left(A_{1}, 1\right) \rightarrow K\left(A_{2}, 1\right)$ restricts to

$$
\bar{\varphi}^{(2)}: K\left(A_{1}, 1\right)^{(2)} \rightarrow K\left(A_{2}, 1\right)^{(2)} .
$$

We show an existence of the induced map $\tilde{\varphi}: X_{1} \rightarrow X_{2}$ of corresponding Moore spaces $X_{1}, X_{2}$ of types $\left(A_{1}, 1\right),\left(A_{2}, 1\right)$, respectively. Let $\left\{a_{\mu}\right\}_{\mu \in I_{1}}$ be a basis of $H_{2}\left(K\left(A_{1}, 1\right)^{(2)}, \mathbb{Z}\right)$. Then the map $f_{\mu}: \mathbb{S}^{2} \rightarrow K\left(A_{1}, 1\right)^{(2)}$ determined by $a_{\mu}$ implies $\bar{\varphi}^{(2)} f_{\mu}: \mathbb{S}^{2} \rightarrow K\left(A_{2}, 1\right)^{(2)}$ for $\mu \in I_{1}$. Next,

$$
\left(\bar{\varphi}^{(2)} f_{\mu}\right)_{*}: H_{2}\left(\mathbb{S}^{2}, \mathbb{Z}\right) \rightarrow H_{2}\left(K\left(A_{2}, 1\right)^{(2)}, \mathbb{Z}\right)
$$

yields an element

$$
b=\left(\bar{\varphi}^{(2)} f_{\mu}\right)_{*}(\gamma) \in H_{2}\left(K\left(A_{2}, 1\right)^{(2)}, \mathbb{Z}\right) .
$$

Since $H_{2}\left(K\left(A_{2}, 1\right)^{(2)}, \mathbb{Z}\right)$ is a free abelian group with a basis $\left\{b_{\lambda}\right\}_{\lambda \in I_{2}}$, we get an isomorphism

$$
H_{2}\left(K\left(A_{2}, 1\right)^{(2)}, \mathbb{Z}\right) \approx \pi_{2}\left(\bigvee_{\lambda \in J} \mathbb{S}^{2}\right)
$$

If $g_{\mu}: \mathbb{S}^{2} \rightarrow \bigvee_{\lambda \in I_{2}} \mathbb{S}^{2}$ is a map corresponding to

$$
b=\left(\bar{\varphi}^{(2)} f_{\mu}\right)_{*}(\gamma) \in \pi_{2}\left(\bigvee_{\lambda \in I_{2}} \mathbb{S}^{2}\right)
$$


then we get a map

$$
\bigvee_{\mu \in I_{1}} g_{\mu}: \bigvee_{\mu \in I_{1}} \mathbb{S}^{2} \longrightarrow \bigvee_{\lambda \in I_{2}} \mathbb{S}^{2}
$$

and the associated map of cones

$$
\bigvee_{\mu \in I_{1}} C\left(g_{\mu}\right): \bigvee_{\mu \in I_{1}} \mathbb{D}^{3} \longrightarrow \bigvee_{\lambda \in I_{2}} \mathbb{D}^{3} .
$$

Consequently, those maps lead the required map $\tilde{\varphi}: X_{1} \rightarrow X_{2}$ with $H_{1}(\tilde{\varphi})=\varphi$. If the spaces $X_{1}, X_{2}$ are nilpotent then Theorem 1.10 implies that $\tilde{\varphi}: X_{1} \rightarrow X_{2}$ is a homotopy equivalence.

If $X_{1}, X_{2}$ are Moore spaces of type $(A, 1)$ constructed in [25] then, by the above, the identity $\operatorname{id}_{A}: A \rightarrow A$ yields a map $\widetilde{\mathrm{id}_{A}}: X_{1} \rightarrow X_{2}$ with $H_{1}\left(\widetilde{\mathrm{id}_{A}}\right)=\operatorname{id}_{A}$ and the proof is complete.

However, the homotopy type of a $C W$-complex Moore space $M(A, 1)$ is not uniquely determined by $A$. Hatcher [15, Example 4.35] constructed the space $X=\left(\mathbb{S}^{1} \vee \mathbb{S}^{n}\right) \cup e^{n+1}$ such that the inclusion $\mathbb{S}^{1} \hookrightarrow X$ induces an isomorphism on all homology groups and on $\pi_{k}$ for $k<n$, but not on $\pi_{n}$. More precisely, from [15, Example 4.27] we have

$$
\pi_{n}\left(\mathbb{S}^{1} \vee \mathbb{S}^{n}\right) \approx \mathbb{Z}\left[t, t^{-1}\right] /(2 t-1) .
$$

Then, $X$ is obtained from $\mathbb{S}^{1} \vee \mathbb{S}^{n}$ by attaching a cell $e^{n+1}$ via a map $\mathbb{S}^{n} \rightarrow \mathbb{S}^{1} \vee \mathbb{S}^{n}$ corresponding to $2 t-1 \in \mathbb{Z}\left[t, t^{-1}\right]$.

Moore spaces of type $(\mathbf{A}, \mathbf{n})$ with $\mathbf{n} \geqslant \mathbf{2}$. To examine the homotopy nilpotency of $M(A, n)$ with $\geqslant 2$, we notice that Proposition 1.11 yields

Corollary 2.2. If $n \geqslant 2$, then

$$
\operatorname{nil} \Omega\left(M\left(\mathbb{Z}_{p^{m}}, n\right)\right)=\infty
$$

for $m=1,2, \ldots \infty$ and $n \geqslant 2$.

Proof. Let $X_{n}(A)=M(A, n)$ with $n \geqslant 2$ or $X_{1}(A)=L(A)$. Then, by the Universal Coefficient Theorem, we have

$$
\tilde{H}_{k}\left(X_{n}\left(\mathbb{Z}_{p^{m}}\right), \mathbb{F}_{p}\right) \approx \begin{cases}\mathbb{Z}_{p^{m}} \otimes \mathbb{F}_{p} \approx \mathbb{F}_{p}, & \text { for } k=n, \\ \operatorname{Tor}\left(\mathbb{Z}_{p^{m}}, \mathbb{F}_{p}\right) \approx \mathbb{F}_{p}, & \text { for } k=n+1, \\ 0, & \text { for } k \neq n, n+1,\end{cases}
$$

for $m, n \geqslant 1$.

Since the Moore space $M\left(\mathbb{Z}_{p^{m}}, n\right) \simeq \Sigma X_{n-1}\left(\mathbb{Z}_{p^{m}}\right)$ for $n \geqslant 2$, we can consider the iterated Samelson product

$$
s_{n}:\left(X_{n-1}\left(\mathbb{Z}_{p^{m}}\right)\right)^{\wedge k} \rightarrow \Omega M\left(\mathbb{Z}_{p^{m}}, n\right) \simeq \Omega \Sigma\left(X_{n-1}\left(\mathbb{Z}_{p^{m}}\right)\right) .
$$


Thus, Proposition 1.11 implies that

$$
\operatorname{nil} \Omega\left(M\left(\mathbb{Z}_{p^{m}}, n\right)\right)=\infty
$$

for $m \geqslant 1$ and $n \geqslant 2$.

Since $\mathbb{Z}_{p^{\infty}} \otimes \mathbb{F}_{p}=0$, we have

$$
\tilde{H}_{k}\left(X_{n}\left(\mathbb{Z}_{p \infty}\right), \mathbb{F}_{p}\right) \approx \begin{cases}\operatorname{Tor}\left(\mathbb{Z}_{p^{\infty}}, \mathbb{F}_{p}\right) \approx \mathbb{F}_{p}, & \text { for } k=n+1, \\ 0, & \text { for } k \neq n+1,\end{cases}
$$

for $n \geqslant 1$. Thus, the argument above collapses.

Therefore, we process as follows. Given $n \geqslant 2$ and a prime $p$, consider the mapping telescope $T$ determined by the sequence of maps

$$
\mathbb{S}^{n} \stackrel{p}{\rightarrow} \mathbb{S}^{n} \stackrel{p}{\rightarrow} \mathbb{S}^{n} \stackrel{p}{\rightarrow} \cdots
$$

Recall that $T$ is the union of the mapping cylinders $M_{k}$ with the copies of $\mathbb{S}^{n}$ in $M_{k}$ and $M_{k-1}$ identified for all $k$. Thus, $T$ is the quotient space of the disjoint union

$$
\bigsqcup_{k=1}^{\infty} \mathbb{S}^{n} \times[k, k+1]
$$

in which each point

$$
\left(x_{k}, k+1\right) \in \mathbb{S}^{n} \times[k, k+1]
$$

is identified with

$$
\left(p\left(x_{k}\right), k+1\right) \in \mathbb{S}^{1} \times[k+1, k+2] .
$$

In the mapping telescope $T$, let $T_{m}$ be the union of the first $m$ mapping cylinders. This deformation retracts onto $\mathbb{S}^{n}$ by deformation retracting each mapping cylinder onto its right end in turn. Since the maps $p: \mathbb{S}^{n} \rightarrow \mathbb{S}^{n}$ are cellular, each mapping cylinder is a $C W$-complex and the telescope $T$ is the increasing union of the subcomplexes $T_{m} \simeq \mathbb{S}^{n}$.

If we attach a cell $e^{n+1}$ to the first $\mathbb{S}^{n}$ in $T$ via the identity map of $\mathbb{S}^{n}$, we obtain a space $X$ which is the increasing union of its subspaces $X_{m}=T_{m} \cup e^{n+1}$ being $M\left(\mathbb{Z}_{p^{m}}, n\right)$ 's. Since,

$$
H_{n}(X, \mathbb{Z}) \approx \operatorname{colim}_{m} H_{n}\left(X_{m}, \mathbb{Z}\right)=\operatorname{colim}_{m} \mathbb{Z}_{p^{m}}=\mathbb{Z}_{p^{\infty}}
$$

and $H_{k}(X, \mathbb{Z})=0$ for $k \neq n$, we derive that $X$ is the Moore space of type $\left(\mathbb{Z}_{\left.p^{\infty}, n\right)}\right.$.

Furthermore, $\Omega(X)=\operatorname{colim}_{m} \Omega\left(X_{m}\right)$ implies a homotopy equivalence

$$
\Omega\left(M\left(\mathbb{Z}_{p^{\infty}}, n\right)\right) \simeq \operatorname{colim}_{m} \Omega\left(M\left(\mathbb{Z}_{p^{m}}, n\right)\right) .
$$

Thus, the non-trivial maps

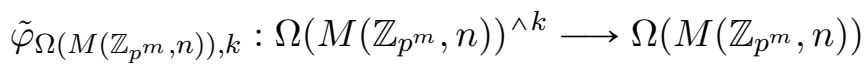


for $k, m \geqslant 1$ determined by (2.1) yield the non-trivial the maps

$$
\tilde{\varphi}_{\Omega\left(M\left(\mathbb{Z}_{p^{\infty}}, n\right)\right), k}: \Omega\left(M\left(\mathbb{Z}_{p^{\infty}}, n\right)\right)^{\wedge k} \longrightarrow \Omega\left(M\left(\mathbb{Z}_{p^{\infty}}, n\right)\right)
$$

for $k \geqslant 1$ and $n \geqslant 2$. Consequently,

$$
\operatorname{nil} \Omega\left(M\left(\mathbb{Z}_{p^{\infty}}, n\right)\right)=\infty
$$

for $n \geqslant 2$ and this concludes the proof.

Now, recall that due to [9, Corollary 27.4], an abelian group $A$ with elements of finite order contains a direct summand $\mathbb{Z}_{p^{m}}$ for some prime $p$ and $m=1,2, \ldots$ or $\infty$. Then, such an abelian group $A \approx \mathbb{Z}_{p^{m}} \oplus B$ for some abelian group $B$ and $m=1, \ldots, \infty$. Hence,

$$
M(A, n)=M\left(\mathbb{Z}_{p^{m}}, n\right) \vee M(B, n),
$$

and therefore (2.1), (2.2), and Remark 1.7 imply that

$$
\operatorname{nil} \Omega(M(A, n))=\infty
$$

for $n \geqslant 2$.

Given an abelian group $A$, we have $A \otimes \mathbb{Q}=\oplus_{1}^{\mathrm{r}(A)} \mathbb{Q}^{+}$for the rank $r(A)$ of $A$ and the additive group $\mathbb{Q}$ of the field $\mathbb{Q}$ of rationals. Therefore,

$$
\tilde{H}_{k}\left(X_{n}(A \otimes \mathbb{Q}), \mathbb{Q}\right) \approx \begin{cases}\bigoplus_{1}^{r(A)} \mathbb{Q}, & \text { for } k=n, \\ 0, & \text { otherwise },\end{cases}
$$

for $n \geqslant 1$.

Next, given a nilpotent space $X$ and a set of primes $I$, write $X_{(I)}$ for the $I$-localization of $X$. Then, by [19, Proposition 3.5], we have

Proposition 2.3. Let $X$ be a nilpotent space. If nil $\Omega(X)<n$ then $\operatorname{nil} \Omega\left(X_{(I)}\right)<n$ for every set of primes $I$.

Since the Moore space $M(A, n) \simeq \Sigma X_{n-1}(A)$ for $n \geqslant 2$, we can consider the iterated Samelson product

$$
s_{n}:\left(X_{n-1}(A)\right)^{\wedge k} \longrightarrow \Omega M(A, n) \simeq \Omega \Sigma\left(X_{n-1}(A)\right) .
$$

Furthermore, if $r(A) \geqslant 2$ then Proposition 1.11 yields

$$
\operatorname{nil} \Omega(M(A \otimes \mathbb{Q}, n))=\infty .
$$

Since $M(A \otimes \mathbb{Q}, n)=M(A, n)_{(0)}$, the rationalization of $M(A, n)$, Proposition 2.3 leads to nil $\Omega(M(A, n))=\infty$. Thus, in view of (1.1), Proposition 1.11 and Corollary 2.2, we may state

Corollary 2.4. Let $A_{1}, \ldots, A_{m}$ be abelian groups and $n_{1}, \ldots, n_{m} \geqslant 2$. 
(1) If $A_{k}$ has an element of finite order or $\mathrm{r}\left(A_{k}\right) \geqslant 2$ for some $k=1, \ldots, m$ then

$$
\operatorname{nil} \Omega\left(\left(M\left(A_{1}, n_{1}\right) \times \cdots \times\left(M\left(A_{m}, n_{m}\right)\right)=\infty\right.\right.
$$

for $m \geqslant 1$.

(2) If $A_{k}$ are non-trivial for $k=1, \ldots, m$ then

$$
\operatorname{nil} \Omega\left(\left(M\left(A_{1}, n_{1}\right) \vee \cdots \vee M\left(A_{m}, n_{m}\right)\right)=\infty\right.
$$

for $m \geqslant 2$.

If $r(A)=1$ and $A$ is a torsion-free abelian group then by [9, Chapter IV, Section 24], we know that $A$ is a subgroup of $\mathbb{Q}$. Notice that

$$
M(\mathbb{Q}, n)=\mathbb{S}_{(0)}^{n}=K(\mathbb{Q}, n),
$$

the Eilenberg-MacLane of type $(\mathbb{Q}, n)$ provided $n$ is odd. Therefore, $M(\mathbb{Q}, n)$ is a homotopy commutative and associative $H$-space and

$$
\operatorname{nil} M(\mathbb{Q}, n)=\operatorname{nil} \Omega(M(\mathbb{Q}, n))=1
$$

Given any subgroup $A<\mathbb{Q}$, we have a sequence $\mathbb{Z} \stackrel{n_{0}}{\rightarrow} \mathbb{Z} \stackrel{n_{1}}{\rightarrow} \mathbb{Z} \stackrel{n_{2}}{\rightarrow} \cdots$ and $A=\operatorname{colim}_{n_{k}} \mathbb{Z}$. Next, for $n \geqslant 2$, the mapping telescope $T$ of the associated sequence of maps

$$
\mathbb{S}^{n} \stackrel{n_{0}}{\rightarrow} \mathbb{S}^{n} \stackrel{n_{1}}{\rightarrow} \mathbb{S}^{n} \stackrel{n_{2}}{\rightarrow} \cdots
$$

is the union of the mapping cylinders $M_{n_{k}}$ with the copies of $\mathbb{S}^{n}$ in $M_{n_{k}}$ and $M_{n_{k-1}}$ identified for all $k$. In the mapping telescope $T$, let $T_{m}$ be the union of the first $m$ mapping cylinders. This deformation retracts onto $\mathbb{S}^{n}$ by deformation retracting each mapping cylinder onto its right end in turn. Since the maps $n_{k}: \mathbb{S}^{n} \rightarrow \mathbb{S}^{n}$ are cellular, each mapping cylinder is a $C W$ complex and the telescope $T$ is the increasing union of the subcomplexes $T_{m} \simeq \mathbb{S}^{n}=M(\mathbb{Z}, n)$. Next, by $\tilde{H}_{n}(T, \mathbb{Z}) \approx A=\operatorname{colim}_{m} \mathbb{Z}$ and $\tilde{H}_{k}(T, \mathbb{Z})=0$ for $k \neq n$, we derive that $T=M(A, n)=\operatorname{colim}_{m} T_{m}$. Then,

$$
\Omega(M(A, n))=\operatorname{colim}_{m} \Omega\left(T_{m}\right), \quad \operatorname{nil} \Omega\left(T_{m}\right)=\operatorname{nil} \Omega\left(\mathbb{S}^{n}\right) \leqslant 3
$$

imply that nil $\Omega(M(A, n)) \leqslant 3$ provided $n \geqslant 2$ and we get the main result

Theorem 2.5. Let $m \geqslant 1, n_{1}, \ldots, n_{m} \geqslant 2$ and $M\left(A_{k}, n_{k}\right)$ be Moore spaces of type $\left(A_{k}, n_{k}\right)$ for $k=1, \ldots, m$. Then

(1) nil $\Omega\left(\left(M\left(A_{1}, n_{1}\right) \times \cdots \times\left(M\left(A_{m}, n_{m}\right)\right)<\infty\right.\right.$ if and only if $A_{k}$ are torsionfree groups with rank $\mathrm{r}\left(A_{k}\right)=1$ for $k=1, \ldots, m$;

(2) nil $\Omega\left(\left(M\left(A_{1}, n_{1}\right) \vee \cdots \vee M\left(A_{m}, n_{m}\right)\right)<\infty\right.$ if and only if $m=1$ and $A_{1}$ is a torsion-free group with rank $\mathrm{r}\left(A_{1}\right)=1$. 
Moore spaces of type $(\mathbf{A}, \mathbf{1})$. We present constructions and analyse homotopy nilpotency of some Moore spaces of type $(A, 1)$.

(1) The space $X=\left(\mathbb{S}^{1} \vee \mathbb{S}^{n}\right) \cup e^{n+1}$ constructed by Hatcher $[15$, Example $4.35]$ is a Moore space of type $(\mathbb{Z}, 1)$ for the infinite cyclic group $\mathbb{Z}\langle t\rangle$. Since

$$
\pi_{1}(X) \approx \mathbb{Z}\langle t\rangle, \quad \quad \pi_{n}\left(\mathbb{S}^{1} \vee \mathbb{S}^{n}\right) \approx \mathbb{Z}\left[t, t^{-1}\right] /(2 t-1),
$$

we get that $(2 t-1) \alpha=0$ implies $2 t \alpha=\alpha$ for $\alpha \in \mathbb{Z}\left[t, t^{-1}\right] /(2 t-1)$. Hence, the action of $\pi_{1}(X)$ on $\pi_{n}(X)$ is non-trivial.

Note that the map $\mathbb{Z}\left[t, t^{-1}\right] \rightarrow \mathbb{Q}$ given by $t \mapsto 1 / 2$ yields a ring isomorphism

$$
\mathbb{Z}\left[t, t^{-1}\right] /(2 t-1) \stackrel{\approx}{\longrightarrow} \mathbb{Z}[1 / 2]
$$

for the subring $\mathbb{Z}[1 / 2] \subseteq \mathbb{Q}$ consisting of rationals with denominator a power of 2 . Then, $\pi_{1}(X) \approx \mathbb{Z}\langle t\rangle$ acts on $\pi_{n}(X) \approx \mathbb{Z}[1 / 2]$ by $t \alpha=(1 / 2) \alpha$ for $\alpha \in \mathbb{Z}[1 / 2]$. Consequently, the Whitehead product $[t, \alpha]=(-1 / 2) \alpha$ and the $(n+1)$-fold Whitehead product $[\alpha, t, t \ldots, t]=(-1 / 2)^{n} \alpha$ is non-trivial provided $\alpha \neq 0$. Therefore in view of Theorem 1.8,

$$
\operatorname{nil} \Omega(X)=\infty \text {. }
$$

(2) The space $\mathbb{R} P_{m}^{2}=\mathbb{S}^{1} \cup_{m} e^{2}$ is a Moore space of type $\left(\mathbb{Z}_{m}, 1\right)$ for the cyclic group $\mathbb{Z}_{m}$ of order $m$. Then, $\pi_{1}\left(\mathbb{R} P_{m}^{2}\right)=\mathbb{Z}_{m}\langle t\rangle$, where $t$ is represented by the canonical map $i: \mathbb{S}^{1} \rightarrow \mathbb{R} P_{n}^{2}$. Next, by [23], the group $\pi_{2}\left(\mathbb{R} P_{m}^{2}\right)$ can be identified with the ideal of the group ring $\mathbb{Z}\left[\mathbb{Z}_{m}\right]$ generated by $\alpha=1-t$, so that as an abelian group $\pi_{2}\left(\mathbb{R} P_{m}^{2}\right)$ is free of rank $m-1$ and, as $\pi_{1}$-module, $\pi_{2}\left(\mathbb{R} P_{m}^{2}\right)$ has a single generator $\alpha$, subject solely to the relation $\left(1+t+\cdots+t^{m-1}\right) \alpha=0$. Then, the Whitehead product

$$
[\alpha, t]=t \alpha-\alpha=2 \alpha-t^{2} \alpha-\cdots-t^{m-1} \alpha .
$$

Thus, we derive that the $(n+1)$-fold Whitehead product $[\alpha, t, t \ldots, t]$ is non-trivial for $n \geqslant 1$. Consequently, in view of Theorem 1.8, we derive that

$$
\operatorname{nil} \Omega\left(\mathbb{R} P_{m}^{2}\right)=\infty
$$

for $m \geqslant 2$.

(3) If $r(A)=1$ and $A$ is a torsion-free abelian group then $A<\mathbb{Q}$, we have a sequence $\mathbb{Z} \stackrel{n_{0}}{\rightarrow} \mathbb{Z}^{n} \stackrel{n_{1}}{\rightarrow} \mathbb{Z} \stackrel{n_{2}}{\rightarrow} \cdots$ and $A=$ colim $_{k_{i}} \mathbb{Z}$. Next, the mapping telescope $T$ of the associated sequence of maps

$$
\mathbb{S}^{1} \stackrel{n_{0}}{\rightarrow} \mathbb{S}^{1} \stackrel{n_{1}}{\rightarrow} \mathbb{S}^{1} \stackrel{n_{2}}{\rightarrow} \ldots
$$

is the union of the mapping cylinders $M_{n_{k}}$ with the copies of $\mathbb{S}^{1}$ in $M_{n_{k}}$ and $M_{n_{k-1}}$ identified for all $k$. In the mapping telescope $T$, let $T_{m}$ be the union of the first $m$ mapping cylinders. This deformation retracts onto $\mathbb{S}^{1}$ by deformation retracting each mapping cylinder onto its right end in turn. 
Since the maps $n_{k}: \mathbb{S}^{1} \rightarrow \mathbb{S}^{n}$ are cellular, each mapping cylinder is a $C W$ complex and the telescope $T$ is the increasing union of the subcomplexes $T_{m} \simeq \mathbb{S}^{1}$.

Next, from

$$
\tilde{H}_{1}(T, \mathbb{Z}) \approx \operatorname{colim}_{m} H_{1}\left(T_{m}\right)=\operatorname{colim}_{k_{i}} \mathbb{Z}=A, \quad \tilde{H}_{k}(T, \mathbb{Z})=0, k \neq 1,
$$

we derive that $T=\operatorname{colim}_{m} T_{m}$ is a Moore space of type $(A, 1)$. Furthermore, $\pi_{k}(T)=\operatorname{colim}_{m} \pi_{k}\left(T_{m}\right)$ implies that $\pi_{1}(T)=\operatorname{colim}_{m} \mathbb{Z}=A$ and $\pi_{k}(T)=0$ for $k \neq 1$. Consequently, $T=\operatorname{colim}_{m} T_{m}$, as the Eilenberg-MacLane space $K(A, 1)$, is a homotopy commutative and an associative $H$-space. Finally, we get that

$$
\operatorname{nil} T=1
$$

(4) At the end of this subsection, given a prime $p$, consider the telescope $T$ determined by the sequence of maps

$$
\mathbb{S}^{1} \stackrel{p}{\rightarrow} \mathbb{S}^{1} \stackrel{p}{\rightarrow} \mathbb{S}^{1} \stackrel{p}{\rightarrow} \cdots .
$$

If we attach a cell $e^{2}$ to the first $\mathbb{S}^{1}$ in $T$ via the identity map of $\mathbb{S}^{1}$, we obtain a space $X$ which is a Moore space of type $\left(\mathbb{Z}_{p^{\infty}}, 1\right)$ since $X$ is the increasing union of its subspaces $X_{m}=T_{m} \cup e^{2}$, which are $\mathbb{R} P_{p^{m}}^{2}$ 's. Then, (2) leads to

$$
\operatorname{nil} \Omega(X)=\infty \text {. }
$$

To conclude, notice that by Seifert-Van Kampen Theorem

$$
\pi_{1}\left(M\left(A_{1}, 1\right) \vee M\left(A_{2}, 1\right)\right)=A_{1} * A_{2},
$$

the free product of $A_{1}$ and $A_{2}$. Furthermore, if $n \geqslant 2$ then

$$
\begin{aligned}
& \pi_{1}\left(M\left(A_{1}, 1\right) \vee M\left(A_{2}, n\right)\right)=A_{1}, \\
& \pi_{n}\left(M\left(A_{1}, 1\right) \vee M\left(A_{2}, n\right)\right) \approx \mathbb{Z}\left[A_{1}\right] \otimes A_{2}
\end{aligned}
$$

for the group ring $\mathbb{Z}\left[A_{1}\right]$. Therefore, the spaces $M\left(A_{1}, 1\right) \vee M\left(A_{2}, n\right)$ for $n \geqslant 1$ are non-nilpotent. Then, Remark 1.7 and Proposition 1.11 yield

Corollary 2.6. If $m \geqslant 2, n_{1}, \ldots, n_{m} \geqslant 1$ and $A_{k}$ are non-trivial Ablian group for $k=1, \ldots, m$ then nil $\Omega\left(\left(M\left(A_{1}, n_{1}\right) \vee \cdots \vee M\left(A_{m}, n_{m}\right)\right)=\infty\right.$.

Smash product of Moore spaces. Recall that the smash product of pointed spaces $X, Y$ is the quotient space

$$
X \wedge Y=(X \times Y) /(X \vee Y)
$$

of the product space $X \times Y$ by the wedge sum $X \vee Y$. It is well-known that the connectivity

$$
\operatorname{conn}(X \wedge Y) \geqslant \operatorname{conn}(X)+\operatorname{conn}(Y)+1 .
$$


Since $\operatorname{conn}(M(A, n))=n-1$ for $n \geqslant 1$, we deduce that

$$
\operatorname{conn}(M(A, m) \wedge M(B, n)) \geqslant m+n-1 \geqslant 1
$$

for $m, n \geqslant 1$. Furthermore, by the Künneth Theorem, we have

$$
\tilde{H}_{k}(M(A, m) \wedge M(B, n), \mathbb{Z})= \begin{cases}A \otimes B, & \text { for } k=m+n, \\ \operatorname{Tor}(A, B), & \text { for } k=m+n+1, \\ 0, & \text { otherwise }\end{cases}
$$

Notice that the rationalization

$(M(A, m) \wedge M(B, n))_{(0)}=M(A \otimes B \otimes \mathbb{Q}, m+n)=M\left(\bigoplus_{1}^{\mathrm{r}(A) \mathrm{r}(B)} \mathbb{Q}, m+n\right)$.

Consequently, Propositions 1.11 and 2.3 yield that

$$
\operatorname{nil} \Omega(M(A, m) \wedge M(B, n))=\infty
$$

provided $\max \{\mathrm{r}(A), \mathrm{r}(B)\} \geqslant 2$.

To analyse the case $\max \{\mathrm{r}(A), \mathrm{r}(B)\} \leqslant 1$ and $\max \{m, n\} \geqslant 2$, we need

Lemma 2.7. For any abelian groups $A$ and $B$, the group $\operatorname{Tor}(A, B)$ is torsion.

Proof. Certainly, we can assume that $A$ and $B$ are torsion groups. We then form an exact sequence

$$
0 \rightarrow \operatorname{ker} \rightarrow \underset{0 \neq b \in B}{\bigoplus} \mathbb{Z}_{|b|} \rightarrow B \rightarrow 0 .
$$

where $|b|$ is the order of $b \in B$ and the map to $B$ is given by sending a generator of the factor corresponding to $b$ to the element $b$. If we write $C$ for the group in the middle, we obtain from the exact sequence for Tor the short exact sequence

$$
\begin{aligned}
0 \rightarrow \operatorname{coker}(\operatorname{Tor}(A, \operatorname{ker}) \rightarrow & \operatorname{Tor}(A, C)) \rightarrow \\
& \rightarrow \operatorname{Tor}(A, B) \rightarrow \operatorname{ker}(A \otimes \operatorname{ker} \rightarrow A \otimes C) \rightarrow 0 .
\end{aligned}
$$

We have that

$$
\operatorname{Tor}(A, C)=\bigoplus_{0 \neq b \in B} \operatorname{Tor}\left(A, \mathbb{Z}_{|b|}\right)=\bigoplus_{0 \neq b \in B} \operatorname{ker}(A \stackrel{|b|}{\rightarrow} A)
$$

and $A \otimes$ ker are torsion groups because $A$ is a torsion group. Hence the first and third term of the sequence above are torsion. The result now follows from the general result that if in an exact sequence $1 \rightarrow G^{\prime} \rightarrow G \rightarrow G^{\prime \prime} \rightarrow 1$ the groups $G^{\prime}$ and $G^{\prime \prime}$ are torsion, then so is $G$ and the proof is complete. 
Since $\max \{m, n\} \geqslant 2$, the smash product $M(A, m) \wedge M(B, n)$ is a suspension. For $r(A)=0$ or $r(B)=0$, the groups $A \otimes B$ and $\operatorname{Tor}(A, B)$ (Lemma 2.7) are torsion. If $A \otimes B \neq 0$ or $\operatorname{Tor}(A, B) \neq 0$ then Proposition 1.11 implies that

$$
\operatorname{nil} \Omega(M(A, m) \wedge M(B, n))=\infty .
$$

If $A \otimes B=0$ and $\operatorname{Tor}(A, B)=0$ then $M(A, m) \wedge M(B, n)$ is an acyclic space. Since the space $M(A, m) \wedge M(B, n)$ has the homotopy type of a $C W$ complex with the trivial fundamental group, the Whitehead and Hurewicz Theorems yield the its contractibility and so

$$
\operatorname{nil} \Omega(M(A, m) \wedge M(B, n))=0 .
$$

Next, $\mathrm{r}(A)=\mathrm{r}(B)=1$ implies $\mathrm{r}(A \otimes B)=1$. If $A \otimes B$ is a mixed group or $\operatorname{Tor}(A, B) \neq 0$ then Proposition 1.11 yields

$$
\operatorname{nil} \Omega(M(A, m) \wedge M(B, n))=\infty .
$$

If $A \otimes B$ is torsion-free and $\operatorname{Tor}(A, B)=0$ then

$$
\operatorname{nil} \Omega(M(A, m) \wedge M(B, n))<\infty .
$$

Now, let $m=n=1$ and write

$$
T(A)=\bigoplus_{p \in I_{A}} T_{p}(A), \quad T(B)=\bigoplus_{p \in I_{B}} T_{p}(B)
$$

for primary decompositions of the subgroups of $T_{p}(A)<A$ and $T_{p}(B)<B$, respectively. But, if $2 \notin I_{A} \cap I_{B}$ then

$$
\pi_{3}(M(\operatorname{Tor}(A, B), 2))=(\operatorname{Tor}(A, B)) \otimes \mathbb{Z}_{2}=0 .
$$

Consequently, by the Universal Coefficient Theorem for Homotopy Groups ([1, Theorem 2.5.9] and [16, Chpter 5]) there is a short exact sequence:

$$
\begin{aligned}
0 \rightarrow \operatorname{Ext}(A \otimes B, & \left.\pi_{3}(M(\operatorname{Tor}(A, B), 2))\right) \\
\rightarrow[M(A \otimes B, 2), M( & \operatorname{Tor}(A, B), 2)] \rightarrow \\
& \rightarrow \operatorname{Hom}(A \otimes B, \operatorname{Tor}(A, B)) \rightarrow 0,
\end{aligned}
$$

It follows that

$$
M(A \otimes B, 2) \rightarrow M(\operatorname{Tor}(A, B), 2)
$$

is the only homology trivial map. Thus, from the homology decomposition (see [16, Chapter 8] for details) of $M(A, 1) \wedge M(B, 1)$, we get that

$$
M(A, 1) \wedge M(B, 1)=M(A \otimes B, 2) \vee M(\operatorname{Tor}(A, B), 3)
$$

is a suspension and so Proposition 1.11 yields

$$
\operatorname{nil} \Omega(M(A, 1) \wedge M(B, 1))<\infty
$$


if and only if the group $A \otimes B$ is torsion free with $\mathrm{r}(A \otimes B) \leqslant 1$ and $\operatorname{Tor}(A, B)=0$.

To examine the case $2 \in I_{A} \cap I_{B}$, we begin from the following situations.

(I) If $k \geqslant 2$ then in view of [22, Proposition 6.2.2], we have

$$
M\left(\mathbb{Z}_{2^{k}}, 1\right) \wedge M\left(\mathbb{Z}_{2^{k}}, 1\right)=M\left(\mathbb{Z}_{2^{k}}, 2\right) \vee M\left(\mathbb{Z}_{2^{k}}, 3\right) .
$$

Hence, Proposition 1.11 leads to

$$
\operatorname{nil} \Omega\left(M\left(\mathbb{Z}_{2^{k}}, 1\right) \wedge M\left(\mathbb{Z}_{2^{k}}, 1\right)\right)=\infty .
$$

(II) If $k=1$ then, by the short exact sequence [1, Theorem 2.5.9], there is the short exact sequence

$$
0 \rightarrow \operatorname{Ext}\left(\mathbb{Z}_{2}, \pi_{3}\left(M\left(\mathbb{Z}_{2}, 2\right)\right)\right) \rightarrow\left[M\left(\mathbb{Z}_{2}, 2\right), M\left(\mathbb{Z}_{2}, 2\right)\right] \rightarrow \operatorname{Hom}\left(\mathbb{Z}_{2}, \mathbb{Z}_{2}\right) \rightarrow 0
$$

with

$$
\begin{gathered}
\operatorname{Ext}\left(\mathbb{Z}_{2}, \pi_{3}\left(M\left(\mathbb{Z}_{2}, 2\right)\right)\right) \approx \mathbb{Z}_{2}, \\
{\left[M\left(\mathbb{Z}_{2}, 2\right), M\left(\mathbb{Z}_{2}, 2\right)\right]=\left[\Sigma \mathbb{R} P^{2}, \Sigma \mathbb{R} P^{2}\right] \approx \mathbb{Z}_{4} .}
\end{gathered}
$$

But, $M\left(\mathbb{Z}_{2}, 1\right)=\mathbb{R} P^{2}$ and $\left[\mathbb{R} P^{2}, \mathbb{R} P^{2}\right] \approx \mathbb{Z}_{2}$. Then, the suspension map $\Sigma \alpha: M\left(\mathbb{Z}_{2}, 2\right) \rightarrow M\left(\mathbb{Z}_{2}, 2\right)$ is homology trivial for the only non-trivial map $\alpha: \mathbb{R} P^{2} \rightarrow \mathbb{R} P^{2}$. Thus,

$$
\mathbb{R} P^{2} \wedge \mathbb{R} P^{2} \simeq \Sigma\left(C \mathbb{R} P^{2} \cup_{\alpha} \mathbb{R} P^{2}\right)
$$

and Proposition 1.11 yields that

$$
\operatorname{nil} \Omega\left(\mathbb{R} P^{2} \wedge \mathbb{R} P^{2}\right)=\infty .
$$

From now on, we restrict to Moore spaces of type $(A, 1)$ constructed in [25]. If $2 \in I_{A} \cap I_{B}$ then, in view of [9, Corollary 27.4], $A=\mathbb{Z}_{2^{k}} \oplus A^{\prime}$ and $B=\mathbb{Z}_{2^{l}} \oplus B^{\prime}$ with some $k, l=1, \ldots, \infty$. Hence, by Proposition 2.1, we get a homotopy retract

$$
M\left(\mathbb{Z}_{2^{k}}, 1\right) \wedge M\left(\mathbb{Z}_{2^{l}}, 1\right) \rightarrow M(A, 1) \wedge M(B, 1) \rightarrow M\left(\mathbb{Z}_{2^{k}}, 1\right) \wedge M\left(\mathbb{Z}_{2^{l}}, 1\right) .
$$

Next, notice that the canonical inclusions

$$
\mathbb{Z}_{2^{\min \{k, l\}}} \hookrightarrow \mathbb{Z}_{2^{k}}, \quad \quad \mathbb{Z}_{2^{\min \{k, l\}}} \hookrightarrow \mathbb{Z}_{2^{l}}
$$

yield a homology isomorphism

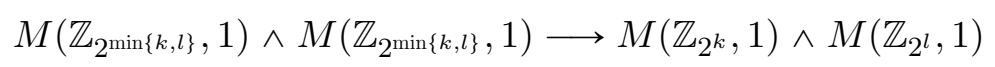

of 1-connected spaces. Thus, Whitehead Theorem leads to a homotopy equivalence

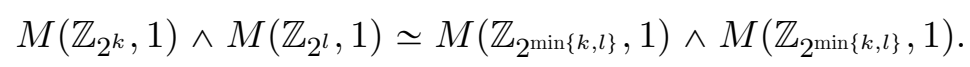

Now, we analyse the following cases. 
(1) If $k=\infty$ or $l=\infty$ then

$$
M\left(\mathbb{Z}_{2^{k}}, 1\right) \wedge M\left(\mathbb{Z}_{2^{l}}, 1\right)=M\left(\mathbb{Z}_{2^{\infty}}, 3\right) .
$$

Since, nil $\Omega\left(M\left(\mathbb{Z}_{2^{\infty}}, 3\right)\right)=\infty$, Remark 1.7 implies

$$
\operatorname{nil} \Omega(M(A, 1) \wedge M(B, 1))=\infty .
$$

(2) If $2 \leqslant \min \{k, l\}<\infty$ then

$$
M\left(\mathbb{Z}_{2^{k}}, 1\right) \wedge M\left(\mathbb{Z}_{2^{l}}, 1\right) \simeq M\left(\mathbb{Z}_{2^{\min \{k, l\}}}, 1\right) \wedge M\left(\mathbb{Z}_{2^{\min \{k, l\}}}, 1\right),
$$

Remark 1.7 and (I) yield

$$
\operatorname{nil} \Omega(M(A, 1) \wedge M(B, 1))=\infty .
$$

(3) If $\min \{k, l\}=1$ then a homotopy equivalence

$$
M\left(\mathbb{Z}_{2^{k}}, 1\right) \wedge M\left(\mathbb{Z}_{2^{l}}, 1\right) \simeq M\left(\mathbb{Z}_{2}, 1\right) \wedge M\left(\mathbb{Z}_{2}, 1\right),
$$

Remark 1.7 and (II) lead to

$$
\operatorname{nil} \Omega(M(A, 1) \wedge M(B, 1))=\infty .
$$

Summarizing the discussion above, we get

Proposition 2.8. If $m, n \geqslant 1$ then

$$
\operatorname{nil} \Omega(M(A, m) \wedge M(B, n))<\infty
$$

if and only if the group $A \otimes B$ is torsion free with the $\operatorname{rank} \mathrm{r}(A \otimes B) \leqslant 1$ and $\operatorname{Tor}(A, B)=0$.

Homology spheres. Recall that a map $f: X \rightarrow Y$ is called a homology isomorphism if it induces isomorphisms on all integral homology groups. Then, according to [7] a space $\mathcal{S}^{n}$ is called a homology $n$-sphere if there is an isomorphism of homologies $H_{k}\left(\mathcal{S}^{n}\right) \approx H_{k}\left(\mathbb{S}^{n}\right)$ for all $k \geqslant 0$. Given a homology $n$-sphere $\mathcal{S}^{n}$ with $n \geqslant 1$, the fundamental group $\pi_{1}\left(\mathcal{S}^{n}\right)$ is perfect for $n \geqslant 2$ and the abelianization $\pi_{1}\left(\mathcal{S}^{1}\right)^{\text {ab }} \approx \mathbb{Z}$, the infinite cyclic group. Thus, the result of Bousfield-Kan [5, Chapter VII, Proposition 3.2, p. 206] implies that there is a homology isomorphism $\mathfrak{h}_{n}: \mathcal{S}^{n} \rightarrow \mathbb{S}^{n}$ for $n \geqslant 2$. It is not difficult to show that there is also a homology isomorphism $\mathfrak{h}_{1}: \mathcal{S}^{1} \rightarrow \mathbb{S}^{1}$.

Remark 2.9. Let $\mathcal{S}^{n}$ be a homology $n$-sphere with $n \geqslant 1$ and $\mathfrak{h}_{n}: \mathcal{S}^{n} \rightarrow \mathbb{S}^{n}$ a homology isomorphism.

(1) Since the fundamental group $\pi_{1}\left(\mathcal{S}^{n}\right)$ is perfect for $n \geqslant 2$, we get that nil $\pi_{1}\left(\mathcal{S}^{n}\right)=\infty$ provided $\pi_{1}\left(\mathcal{S}^{n}\right) \neq E$. Thus, nil $\pi_{1}\left(\mathcal{S}^{n}\right) \leqslant \operatorname{nil} \Omega\left(\mathcal{S}^{n}\right)$ implies that nil $\Omega\left(\mathcal{S}^{n}\right)=\infty$ provided $\pi_{1}\left(\mathcal{S}^{n}\right) \neq E$. 
(2) Since a homology $n$-sphere $\mathcal{S}^{n}$ is path-connected, the homology isomorphism $\mathfrak{h}_{n}: \mathcal{S}^{n} \rightarrow \mathbb{S}^{n}$ leads to a weak homotopy equivalence

$$
\Sigma\left(\mathfrak{h}_{n}\right): \Sigma\left(\mathcal{S}^{n}\right) \rightarrow \Sigma\left(\mathbb{S}^{n}\right)=\mathbb{S}^{n+1}, \quad n \geqslant 1 .
$$

In particular, $\Sigma\left(\mathcal{S}^{n}\right)$ has the homotopy type of the sphere $\mathbb{S}^{n+1}$ provided $\mathcal{S}^{n}$ is a $C W$-complex. This implies that

$$
\operatorname{nil} \Omega\left(\Sigma\left(\mathcal{S}^{n}\right)\right)=\operatorname{nil} \Omega\left(\mathbb{S}^{n+1}\right) \leqslant 3, \quad n \geqslant 1 .
$$

(3) If $X, Y$ are $C W$-complexes then the connectivity of the smash product satisfies the following inequality:

$$
\operatorname{conn}(X \wedge Y) \geqslant \operatorname{conn}(X)+\operatorname{conn}(Y)+1
$$

(if both $X$ and $Y$ are connected).

Thus, $\mathfrak{h}_{m}: \mathcal{S}^{m} \rightarrow \mathbb{S}^{m}$ and $\mathfrak{h}_{n}: \mathcal{S}^{n} \rightarrow \mathbb{S}^{n}$ induce a homotopy equivalence

$$
\mathfrak{h}_{m} \wedge \mathfrak{h}_{n}: \mathcal{S}^{m} \wedge \mathcal{S}^{n} \rightarrow \mathbb{S}^{m} \wedge \mathbb{S}^{n}=\mathbb{S}^{m+n}
$$

provided that $\mathcal{S}^{m}$ and $\mathcal{S}^{n}$ are connected $C W$-complexes.

(4) Since the fundamental group of a co- $H$-space is free, a co- $H$-structure on $\mathcal{S}^{n}$ implies that $\mathcal{S}^{n}$ is 1 -connected for $n \geqslant 2$ and $\pi_{1}\left(\mathcal{S}^{1}\right) \approx \mathbb{Z}$. Consequently, $\mathfrak{h}_{n}: \mathcal{S}^{n} \rightarrow \mathbb{S}^{n}$ is a weak homotopy equivalence for $n \geqslant 1$, provided $\mathcal{S}^{n}$ has a co- $H$-structure.

Let $M^{n}$ be a smooth homology $n$-sphere, i.e., a smooth $n$-dimensional manifold such that $H_{*}\left(M^{n}\right) \approx H_{*}\left(\mathbb{S}^{n}\right)$. Then, by Kervire [18], the fundamental group $\pi=\pi_{1}\left(M^{n}\right)$ of $M^{n}$ satisfies the following three conditions:

(1) $\pi$ as a finite presentation,

(2) $H_{1}(\pi)=0$

(3) $H_{2}(\pi)=0$

where $H_{k}(\pi)$ denotes the $k$-th homology group of $\pi$ with coefficients in the trivial $\mathbb{Z}[\pi]$-module $\mathbb{Z}$. Concretely, this means that the abelianization of $\pi$ is trivial (it has no non-trivial abelian quotients) and that its Schur multiplier is trivial (it has no non-trivial perfect central extensions).

Furthermore, by [18, Theorem 2], we have

Theorem 2.10. Let $M^{3}$ be a 3-dimensional manifold such that $\pi_{1}\left(M^{3}\right)$ is finite and $H_{*}\left(M^{3}\right) \approx H_{*}\left(\mathbb{S}^{3}\right)$. Then, either $\pi_{1}\left(M^{3}\right)=E$ or else, $\pi_{1}\left(M^{3}\right)$ is isomorphic to the binary icosahedral group with presentation

$$
\left\langle x, y: x^{2}=y^{3}=(x y)^{5}\right\rangle .
$$

The Poincarè homology sphere (also known as Poincarè dodecahedral space) is a particular example of a smooth homology sphere, first constructed by Henri Poincaré. Being a spherical 3-manifold, it is the only 
homology 3 -sphere (besides the 3 -sphere itself) with a finite fundamental group.

Alternatively, the Poincarè homology sphere can be constructed as the quotient space $S O(3) / I$, where $S O(3)$ is the special orthogonal group in dimension 3 and $I$ is the icosahedral group (i.e., the rotational symmetry group of the regular icosahedron and dodecahedron, isomorphic to the alternating group $A_{5}$ ).

One can also pass instead to the universal cover of $S O(3)$ which can be realized as the group of unit quaternions and is homeomorphic to the 3 -sphere $\mathbb{S}^{3}$. In this case, the Poincarè homology sphere is isomorphic to $\mathbb{S}^{3} / \tilde{I}$, where $\tilde{I}$ is the binary icosahedral group, the perfect double cover of $I$ embedded in $\mathbb{S}^{3}$.

Consequently, nil $\Omega\left(\mathbb{S}^{3} / \tilde{I}\right)=\infty$, whereas nil $\Omega\left(\Sigma\left(\mathbb{S}^{3} / \tilde{I}\right)\right)=\operatorname{nil} \Omega\left(\mathbb{S}^{4}\right)=$ 3.

\section{REFERENCES}

[1] Martin Arkowitz. Introduction to homotopy theory. Universitext. Springer, New York, 2011, doi: 10.1007/978-1-4419-7329-0.

[2] Martin Arkowitz, Marek Golasiński. Co- $H$-structures on Moore spaces of type $(G, 2)$. Canad. J. Math., 46(4):673-686, 1994, doi: 10.4153/CJM-1994-037-0.

[3] I. Berstein, T. Ganea. Homotopical nilpotency. Illinois J. Math., 5:99-130, 1961, http: //projecteuclid.org/euclid.ijm/1255629648.

[4] R. Bott, H. Samelson. On the Pontryagin product in spaces of paths. Comment. Math. Helv., 27:320-337 (1954), 1953, doi: 10.1007/BF02564566.

[5] A. K. Bousfield, D. M. Kan. Homotopy limits, completions and localizations. Lecture Notes in Mathematics, Vol. 304. Springer-Verlag, Berlin-New York, 1972.

[6] Ethan S. Devinatz, Michael J. Hopkins, Jeffrey H. Smith. Nilpotence and stable homotopy theory. I. Ann. of Math. (2), 128(2):207-241, 1988, doi: 10.2307/1971440.

[7] Emmanuel Dror. A generalization of the Whitehead theorem. Lecture Notes in Math., 249:13-22, 1971.

[8] Emmanuel Dror. Homology spheres. Israel J. Math., 15:115-129, 1973, doi: $10.1007 / \mathrm{BF} 02764597$.

[9] László Fuchs. Infinite abelian groups. Vol. I. Pure and Applied Mathematics, Vol. 36. Academic Press, New York-London, 1970.

[10] Tudor Ganea. Lusternik-Schnirelmann category and cocategory. Proc. London Math. Soc. (3), 10:623-639, 1960, doi: 10.1112/plms/s3-10.1.623.

[11] Marek Golasiński. A survey of the homotopy nilpotency and co-nilpotency. Proc. Int. Geom. Cent., 13(4):1-23, 2020, doi: 10.15673/tmgc.v13i4.1750.

[12] Marek Golasiński. Homotopy nilpotency of localized spheres and projective spaces. Proc. Edinb. Math. Soc. (2), 64(3):501-512, 2021, doi: 10.1017/S0013091521000274.

[13] Marek Golasiński. Homotopy nilpotency of some homogeneous spaces. Manuscripta Math., 2021, doi: 10.1007/s00229-021-01273-y.

[14] Marek Golasiński. On homotopy nilpotency of loop spaces of moore spaces. Canad. Math. Bull., pages 1-12, 2021, doi: 10.4153/S000843952100028X.

[15] Allen Hatcher. Algebraic topology. Cambridge University Press, Cambridge, 2002. 
[16] Peter Hilton. Homotopy theory and duality. Gordon and Breach Science Publishers, New York-London-Paris, 1965.

[17] Michael J. Hopkins. Nilpotence and finite $H$-spaces. Israel J. Math., 66(1-3):238-246, 1989, doi: 10.1007/BF02765895.

[18] Michel A. Kervaire. Smooth homology spheres and their fundamental groups. Trans. Amer. Math. Soc., 144:67-72, 1969, doi: 10.2307/1995269.

[19] Willi Meier. Homotopy nilpotency and localization. Math. Z., 161(2):169-183, 1978, doi: 10.1007/BF01214929.

[20] Clair Miller. The second homology group of a group; relations among commutators. Proc. Amer. Math. Soc., 3:588-595, 1952, doi: 10.2307/2032593.

[21] John C. Moore. On homotopy groups of spaces with a single non-vanishing homology group. Ann. of Math. (2), 59:549-557, 1954, doi: 10.2307/1969718.

[22] Joseph Neisendorfer. Algebraic methods in unstable homotopy theory, volume 12 of New Mathematical Monographs. Cambridge University Press, Cambridge, 2010, doi: 10.1017/CBO9780511691638.

[23] Allan J. Sieradski. Stabilization of self-equivalences of the pseudoprojective spaces. Michigan Math. J., 19:109-119, 1972, http://projecteuclid.org/euclid.mmj/ 1029000840.

[24] Victor P. Snaith. Some nilpotent $H$-spaces. Osaka Math. J., 13(1):145-156, 1976, http: //projecteuclid.org/euclid.ojm/1200769310.

[25] K. Varadarajan. Groups for which Moore spaces $M(\pi, 1)$ exist. Ann. of Math. (2), 84:368-371, 1966, doi: 10.2307/1970450.

[26] George W. Whitehead. On products in homotopy groups. Ann. of Math. (2), 47:460475, 1946, doi: $10.2307 / 1969085$.

[27] George W. Whitehead. Elements of homotopy theory, volume 61 of Graduate Texts in Mathematics. Springer-Verlag, New York-Berlin, 1978.

[28] Alexander Zabrodsky. Hopf spaces. North-Holland Mathematics Studies, Vol. 22. North-Holland Publishing Co., Amsterdam-New York-Oxford, 1976.

Received: June 30, 2021, accepted: Augist 19, 2021.

Marek Golasiński

Faculty of Mathematics and Computer Science, University of Warmia and Mazury, SŁoneczna 54 Street, 10-710 Olsztyn, Poland

Email: marekg@matman.uwm.edu.pl

ORCID: 0000-0001-6969-8986 